\title{
Marktmissbrauchsrecht und Konzernrecht
}

\author{
DÖRTE POELZIG
}

\section{Einleitung}

Klaus J. Hopt hat sich in seinen vielfältigen und grundlegenden Arbeiten zum Kapitalmarktrecht auch immer wieder der Frage gewidmet, welche Rolle Konzerne spielen. So konstatierte er schon im Jahr 2001, dass das „Thema ,Kapitalmarkt und Konzerne' [...] nach deutschem Recht schwierig zu behandeln“ ist. ${ }^{1}$ Die Ergebnisse seines damaligen Beitrags „zeigen einigermaßen schonungslos auf, dass manches im deutschen Kapitalmarktrecht doch noch sehr lückenhaft, um nicht zu sagen, inkohärent geregelt ist “. ${ }^{2}$ Der vorliegende Beitrag für die Festschrift zu Ehren des Jubilars soll Anlass sein, um anhand der Marktmissbrauchsverordnung nach nunmehr fast 20 Jahren einen neuerlichen Blick auf die Frage zu werfen, inwieweit Konzerne im Kapitalmarktrecht verantwortlich zeichnen und in welchem Verhältnis Marktmissbrauchs- und Konzernrecht zueinander stehen. ${ }^{3}$ Konflikte können vor allem auftreten, da das Marktmissbrauchsrecht seit 2016 in der unmittelbar anwendbaren Marktmissbrauchsverordnung (EU) 596/2014 vereinheitlicht wurde, das Konzernrecht von einer Vereinheitlichung auf europäischer Ebene hingegen aber noch immer weit entfernt ist. ${ }^{4}$ Klaus J. Hopt stellte daher bereits im Jahr 2001 die Frage in den Raum, ob die Spannungen zwischen Kapitalmarktrecht und Konzernrecht ,aus europäischer Sicht [...] besser durch Konzernrecht oder durch Kapitalmarktrecht oder durch ein Zusammenwirken von Normen aus beiden Rechtsgebieten" gelöst werden können. ${ }^{5}$ Diese Frage stellt sich heute mehr denn je, ${ }^{6}$ denn im europäischen Recht greifen Tendenzen weit um sich, Konzerne unbeeindruckt von den S. 31 .

${ }^{1}$ HOPT, in: Hommelhoff/Hopt/Lutter, Konzernrecht und Kapitalmarktrecht, 2001,

${ }^{2}$ HOPT, aaO (Fn. 1), S. $31 \mathrm{f}$.

${ }^{3}$ HOPT/KumPan, in: Schimansky/Bunte/Lwowski, Handbuch Bankrecht, 5. Aufl., 2017, \ 107 Rdn. 138; HOPT/KUMPAN, ZGR 2017, 756, 823 ff.

${ }^{4}$ Hierzu Haberack/VERSE, Europäisches Gesellschaftsrecht, 5. Aufl. 2019, § 4 Rn. 34 ff.; TeICHMANN, in Hommelhoff/Lutter/Teichmann, Corporate Governance im grenzüberschreitenden Konzern, 2017, S. 3, 16; TeICHMANN, ZGR 2017, 485, 489.

${ }^{5}$ HOPT, aaO (Fn. 1), S. 31, 33.

${ }^{6}$ Hommelhoff, ZGR 2019, 379 ff.; POelzig, in: VGR (Hrsg.), Gesellschaftsrecht in der Diskussion, 2017, S. 83, 106; TEICHMANN, ZGR 2017, $485 \mathrm{ff}$. 
Grenzen des nationalen Gesellschaftsrechts als Einheitsunternehmen in die Verantwortung zu nehmen. ${ }^{7} \mathrm{Ob}$ und inwieweit das Marktmissbrauchsrecht von diesen Angriffen auf das konzernrechtliche Trennungsprinzip betroffen ist und inwieweit hier Spannungen mit dem Konzernrecht auftreten und gelöst werden, soll im Folgenden näher untersucht werden.

Dazu ist zunächst exemplarisch anhand der Ad-hoc-Informationspflicht einer kapitalmarktorientierten Muttergesellschaft für Rechtsverstöße in Tochtergesellschaften die konzerndimensionale Reichweite des Marktmissbrauchsrechts zu bestimmen (II.). Anschließend gilt es zu untersuchen, ob und inwieweit Konzerne als wirtschaftliche Einheit für marktmissbräuchliches Verhalten bußgeldrechtlich in die Verantwortung genommen werden (können) (III.). Der Beitrag schließt mit zusammenfassenden Thesen (IV.).

\section{Konzernweite Ad-hoc-Publizitätspflicht für Rechtsverstöße in der Tochtergesellschaft}

Gem. Art. 17 Abs. 1 MAR sind Emittenten verpflichtet, sie unmittelbar betreffende Insiderinformationen unverzüglich zu veröffentlichen. Handelt es sich bei dem Emittenten um eine kapitalmarktorientierte Muttergesellschaft, erstreckt die Rechtsprechung die Ad-hoc-Publizitätspflicht mehr oder weniger weitgehend auf Umstände in der Sphäre ihrer Tochtergesellschaften. Beispielhaft hierfür ist die aktuelle Diskussion um die Ad-hocInformationspflicht der Muttergesellschaft für Compliance-Verstöße in der Volkswagen AG rund in der sog. Dieselaffäre. ${ }^{8}$ Ebenso hat der BGH in der IKB-Entscheidung die „Höhe des Subprime-Anteils der unmittelbar eigenen Investments der [IKB] sowie derjenigen der mit der [IKB] verbundenen Zweckgesellschaften " als veröffentlichungspflichtige Insiderinformation eingeordnet. ${ }^{9}$ Die Annahme einer Pflicht zur Veröffentlichung von Umständen in der Tochter kann aber u.U. an gesellschaftsrechtliche Grenzen stoßen, wenn es der Mutter nicht möglich ist, an diese Informationen zu gelangen. Daher stellt sich die Frage, wie das durch die Marktmissbrauchsverordnung geschützte Interesse des Kapitalmarktes an Information und die konzernrechtlichen Grenzen in Ausgleich zu bringen sind. ${ }^{10}$ Art. 17 Abs. 1 MAR enthält bekanntermaßen keine „Konzernklausel“, sodass die materiell-rechtliche Verantwortlichkeit für die Einhaltung der Ad-hoc-Publizi-

7 Hierzu Hommelhoff, ZGR 2019, 379 ff.; Poelzig, aaO (Fn. 6), S. 83 ff.

${ }^{8}$ Siehe LG Stuttgart, Beschl. v. 28.2.2017 - 22 AR 1/17 Kap = WM 2017, 1451. Zur ähnlichen Problematik der konzerndimensionalen Auskunftspflicht gem. \131 AktG LG Stuttgart, Urt. v. 19.12.2017 - 31 O 33/16 KfH = NZG 2018, 665.

9 BGH, Urt. v. 13.12.2011 - XI ZR 51/10, BGHZ 192, 90 Rdn. 36.

10 Eingehend hierzu GÖTZE/CARL, Der Konzern 2016, 529, 530; KOCHER, NZG 2018, 1410. 
tätspflicht nach wie vor ${ }^{11}$ bei den jeweiligen kapitalmarktorientierten Konzerngesellschaften als rechtlich selbständige Personen liegt. ${ }^{12}$ Das folgt für die Ad-hoc-Informationspflicht aus dem Begriff „Emittent“, der gem. Art. 3 Abs. 1 Nr. 21 MAR stets eine juristische Person ist ${ }^{13}$ und demzufolge nicht der Konzern als wirtschaftliche Einheit sein kann. Damit akzeptiert das Marktmissbrauchsrecht grundsätzlich die Aufteilung von wirtschaftlichen Tätigkeiten auf verschiedene rechtliche Einheiten ${ }^{14}$ und unterscheidet sich dadurch wesentlich von dem europäischen Kartellrecht, das in Art. 101, 102 AEUV Unternehmen als wirtschaftliche Einheiten unabhängig von ihrer Rechtsform adressiert. ${ }^{15}$

\section{Unmittelbare Betroffenheit der Muttergesellschaft}

Die Ad-hoc-Informationspflicht der Muttergesellschaft hängt zunächst davon ab, ob das Ereignis in der Tochtersphäre die Muttergesellschaft „unmittelbar betrifft".16 Auch wenn Art. 17 MAR die unmittelbare Betroffenheit nicht mehr beispielhaft damit umschreibt, dass die Information im Tätigkeitsbereich des Emittenten eingetreten ist, ist eine unmittelbare Betroffenheit nach wie vor dann unproblematisch anzunehmen, wenn sich der Umstand im Tätigkeitsbereich der Muttergesellschaft auswirkt. ${ }^{17}$ Die Muttergesellschaft ist daher jedenfalls von Ereignissen unmittelbar betroffen, die bei vollkonsolidierten Tochterunternehmen (\$S $290 \mathrm{ff}$. HGB) auftreten und sich im Konzernabschluss oder -lagebericht bzw. Halbjahresfinanzbericht

11 Hierzu Hopt, aaO (Fn. 1), S. 31, 58.

12 Hopt/Kumpan, aaO (Fn. 3), \$107 Rdn. 138. Siehe bereits HOPT, ZHR 159 (1995), 135, 151; DERS., aaO (Fn. 1), S. 31, 58; FROWEIN, in: Habersack/Mülbert/Schlitt, Handbuch Kapitalmarktinformation, 2. Aufl., 2013, $\$ 10$ Rdn. 9. Lediglich Art. 17 Abs. 2 MAR enthält eine bislang nur wenig beachtete Konzernklausel, wonach die Ad-hoc-Publizitätspflicht für Teilnehmer am Markt für Emissionszertifikate, soweit der Marktteilnehmer selbst, Mutterunternehmen oder verbundene Unternehmen Emissionszertifikate besitzen.

${ }^{13} \mathrm{Zu} \ 15 \mathrm{WpHG}$ aF.HOPT, aaO (Fn. 1), S. 31, 58.

${ }^{14} \mathrm{Vgl}$. SCHÜRNBRAND, ZHR 181 (2017), 357, 361.

${ }^{15}$ Demnach sind Konzerne als wirtschaftliche Einheit zu einem Normadressaten des Kartellrechts verklammert, wenn die Mutter bestimmenden Einfluss auf die Tochter ausüben kann und tatsächlich ausübt, „die Tochtergesellschaft also trotz eigener Rechtspersönlichkeit ihr Marktverhalten nicht selbständig bestimmt, sondern im Wesentlichen Weisungen der Muttergesellschaft befolgt" (ständige Rspr., statt aller EuGH, Urt. v. 27.4.2017 - C 516/15 P = ECLI:EU:C:2017:314, Rz. 52: Akzo Nobel; EuGH, Urt. v. 17.9.2015 - C 597/13 P = ECLI:EU:C:2015:613, Rz. 35: Total SA). Der EuGH vermutet seit seinem Akzo-Urteil aus dem Jahre 2009 das Vorliegen einer solchen wirtschaftlichen Einheit, wenn die Mutter (annähernd) alle Anteile an der Tochter hält (EuGH, Urt. v. 10.9.2009 C 97/08 P = ECLI:EU:C:2009:536, Rz. 60: Akzo Nobel; jetzt ständige Rspr., statt aller: EuGH, Urt. v. 16.6.2016 - C 155/14 P = ECLI:EU:C:2016:446, Rz. 28: Evonik Degussa und $\mathrm{AlzChem})$.

16 KOCHER, NZG 2018, 1410.

17 Siehe HOPT/KUMPAN, ZGR 2017, 765, 824. 
( $\iint 117 \mathrm{iVm} .115 \mathrm{WpHG}$ ) widerspiegeln. ${ }^{18}$ Die Betroffenheit kann aber (ausnahmsweise) auch bei Umständen außerhalb des Tätigkeitsbereichs des Emittenten gegeben sein. ${ }^{19}$ Wann dies im Verhältnis der Mutter- zur Tochtergesellschaft der Fall ist, ist jedoch umstritten. Nach teilweise vertretener Auffassung ist die Mutter unmittelbar betroffen, wenn die Ereignisse in der Tochter den Fundamentalwert der von der Mutter begebenen Finanzinstrumente verändern. ${ }^{20}$ Hierdurch werden allerdings bereits Elemente der Kursrelevanz im Rahmen der unmittelbaren Betroffenheit berücksichtigt. ${ }^{21}$ Andere grenzen den Tätigkeitsbereich wirtschaftlich wertend und nicht formal nach dem Rechtsträger ab, sodass bei entsprechend hoher Beteiligung die Muttergesellschaft von Ereignissen in der Tochtergesellschaft unmittelbar betroffen sein kann. ${ }^{22}$

Für eine im Grundsatz formal auf den Rechtsträger abstellende Perspektive der unmittelbaren Betroffenheit spricht jedoch, dass das Marktmissbrauchsrecht im Allgemeinen und die Ad-hoc-Informationspflicht im Besonderen juristische Personen als rechtliche Einheiten adressieren. Sinn und Zweck der unmittelbaren Betroffenheit ist es, die Veröffentlichungspflicht auf diejenigen Insiderinformationen gem. Art. 7 Abs. 1 MAR zu beschränken, ${ }^{23}$ die dem Verantwortungsbereich des Emittenten so nahestehen, dass ihm die Suche, Analyse und Veröffentlichung als cheapest cost avoider zu den geringstmöglichen Kosten möglich ist, er also über einen Kostenvorteil gegenüber den anderen Marktteilnehmern verfügt. ${ }^{24}$ Von einem Kostenvor-

18 Hopt/Kumpan, aaO (Fn. 3), $\$ 107$ Rdn. 145; DiEs., ZGR 2017, 765, 824; GRUNDMANN, in: Großkomm. z. HGB, Bd. XI Bankvertragsrecht, 6. Teil Rdn. 506; KLÖHN, in: KLÖHN, Marktmissbrauchsverordnung, 2018, Art. 17 MAR Rdn. 97; PFÜLlER, in: Fuchs, Komm. z. WpHG, 2. Aufl., 2016, \15 Rdn. 207; AssmanN, in: Assmann/Schneider/Mülbert, Wertpapierhandelsrecht, 7. Aufl. 2019, Art. 17 MAR Rdn. 49; KUMPAN/ GRÜTZE, in: Schwark/Zimmer, Komm. z. KapitalmarktR, 5. Aufl. 2020, Art. 17 MAR Rdn. 65; auch SIMON, Der Konzern 2005, 13, 16; SPINDLER/SPEIER, BB 2005, 3021.

19 KLÖHN, aaO (Fn. 18), Art. 17 MAR Rdn. 89.

${ }^{20}$ KLÖHN, aaO (Fn. 18), Art. 17 MAR Rdn. 92 f.; anschließend BARTMAnN, Ad-hocPublizität im Konzern, 2017, S. 295 f.

21 GÖTZE/CARL, Der Konzern 2016, 529, 530; HABERSACK, DB 2016, 1551, 1556; VeIL/ BRÜGGEMEIER, in: Meyer/Veil/Rönnau, HdB Marktmissbrauchsrecht, 2018, \10 Rdn. 62.

22 VeIL/BrügGEMEIER, in: Meyer/Veil/Rönnau, aaO (Fn. 21), $₫ 10$ Rdn. 62 f. im Anschluss an PFÜLLER, in: Fuchs, aaO (Fn. 18), \$15 WpHG Rdn. 208.

${ }^{23}$ Siehe auch zur Einführung der gleichlautenden Regelung in Art. 1 Abs. 1 Marktmissbrauchsrichtlinie aF. SEK (2002) 0889 endg. - 2001/0118 (COD), S. 5); zu $\$ 15$ WpHG aF BT-Drucks. 15/3174, S. 35 li.Sp.

${ }^{24}$ HOPT/VOIGT, in: Hopt/Voigt (Hrsg.), Prospekt- und Kapitalmarktinformationshaftung, 2005, S. 9, 113 f.; KÖNDGEN, FS Druey, 2002, S. 791, 796; SEIBT, ZHR 177 (2013), 387, 393; KLÖHN, WM 2010, 1869, 1878; KLÖHN, NZG 2017, 1285, 1287; KLÖHN, in: Klöhn aaO (Fn. 18), Art. 17 MAR Rdn. 69, 108; KLÖHN, in: KölnKomm z. WpHG, 2. Aufl. 2014, $₫ 15$ Rdn. 7, 59; ZETZSCHE, Aktionärsinformation in der börsennotierten Aktiengesellschaft, 2006, S. 37. Grundlegend zur ökonomischen Begründung STIGLER, The Journal of Political Economy 69 (1961), $213 \mathrm{ff}$. 
teil der Mutter ist bei Umständen in ihrem eigenen Tätigkeitsbereich generell auszugehen; bei Umständen in der Tochtersphäre nur dann, wenn der Tätigkeitsbereich der Tochtergesellschaft dem Tätigkeitsbereich der Mutter gleichgestellt werden kann. Daher ist der Emittent jedenfalls von solchen Informationen unmittelbar betroffen, die in Unternehmen auftreten, auf deren unternehmerische Tätigkeit er einen beherrschenden Einfluss ausüben kann. ${ }^{25}$ Dann steht er der Information grundsätzlich näher als der Markt.

\section{Pflicht der Muttergesellschaft zur unverzüglichen Veröffentlichung}

Grenzen der Informationspflicht der Mutter ergeben sich daraus, dass der Emittent unverzüglich, dh. so bald wie objektiv möglich („as soon as possible") zu veröffentlichen hat. ${ }^{26}$ Voraussetzung hierfür ist, dass der Emittent die Information kennt oder sie sich beschaffen kann. Über die Bedeutung der gesellschaftsrechtlichen Grenzen der Informationsbeschaffung für die Ad-hoc-Informationspflicht in Konzernstrukturen wird an dieser Stelle gestritten. Eine konzernweite Ad-hoc-Informationspflicht ist jedenfalls dann nicht ausgeschlossen, wenn die Mutter bereits nach gesellschaftsrechtlichen Grundsätzen über einen gesicherten Informationszugang verfügt, d.h. ihr wie im Falle von $\iint 308$ Abs. 1, 323 Abs. 1 AktG ${ }^{27}$ oder $\int 51 \mathrm{GmbHG}$ - ein Auskunftsanspruch gegen die Tochtergesellschaft zusteht. ${ }^{28}$ Im faktischen Aktienkonzern fehlt es der Mutter hingegen an einem rechtlich abgesicherten Informationszugang. Ihr steht kein allgemeiner über das beschränkte Auskunftsrecht des Aktionärs gem. \131 Abs. 1 AktG hinausgehender Informationsanspruch gegen die Tochter zu. ${ }^{29}$ Der spezielle Auskunftsan-

25 LG Stuttgart, Beschl. v. 28.2.2017 - 22 AR 1/17 Kap = WM 2017, 1451 Rdn. 172; zu $\int 15$ WpHG aF Zimmer/KRUSE, in: Schwark/Zimmer Komm. z. KapitalmarktR, 4. Aufl. 2010, 15 WpHG Rdn. 46.

26 HOPT/KUMPAN ZGR 2017, 765, 782; KLÖHN, NZG 2017, 1285, 1286f.; KLÖHN, in: KLÖHN, aaO (Fn. 18), Art. 17 MAR Rn. 105; HellgARDT, in: Assmann/Schneider/

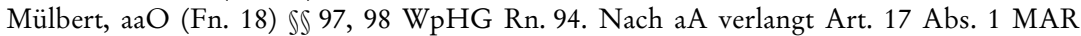
Kenntnis des Emittenten als ein ungeschriebenes Tatbestandsmerkmal (Koch, AG 2019, 273, 276; Assmann, in: Assmann/Schneider/Mülbert, aaO (Fn. 18) Art. 17 MAR Rn. 50; für Wissenserfordernis erst im Rahmen der Haftung gem. \97 WpHG Thomale, Der gespaltene Emittent, 2018, S. $51 \mathrm{ff}$.).

27 HABERSACK, in: FS Möschel, 2011, S. 1175, 1187; LUTTER, in: FS Goette, 2011, S. 289, 293; SCHÜRNBRAND, ZHR 181 (2017), 357, 367; VERSE, ZHR 175 (2011), 401, 418.

28 GÖTZE/CARL, Der Konzern 2016, 529, 530; HABERSACK, in: FS 25 Jahre WpHG, 2019, S. 217, 232; zu \15 WpHG PFÜller, in: Fuchs (Fn. 18), \ 15 Rdn. 206; SPINDLER/SPEIER, BB 2005, 2031, 2032; aA AssmANN, in: Assmann/Schneider/Mülbert, aaO (Fn. 18) Art. 17 MAR Rn. 59.

29 HABERSACK, in: FS Möschel, 2011, S. 1175, 1191; VERSE, ZHR 175 (2011), 401, 423. Allgemein zum Fehlen eines Auskunftsrechts statt aller ALTMEPPEN, in: MüKo-AktG, 
spruch gem. \294 Abs. 3 S. 2 HGB ist auf die Überlassung von Informationen zur Erstellung des Konzernabschlusses beschränkt und ist weder analog anwendbar noch nach seinem Rechtsgedanken verallgemeinerungsfähig. ${ }^{30}$

Dieser Konflikt zwischen dem marktmissbrauchsrechtlichen Interesse an einer optimalen Informationsversorgung des Kapitalmarktes und den konzernrechtlichen Restriktionen wird teilweise zugunsten des Marktmissbrauchsrechts aufgelöst, indem aus Art. 17 Abs. 1 MAR ein allgemeiner Auskunftsanspruch der Mutter gegen die Tochter abgeleitet wird. ${ }^{31}$ Der kapitalmarktrechtliche Auskunftsanspruch wird vor allem damit begründet, dass die Ad-hoc-Informationspflicht anderenfalls durch gezielte konzernrechtliche Organisation umgangen und im Widerspruch zum Effektivitätsgrundsatz ihrer praktischen Wirksamkeit beraubt werden könne. ${ }^{32}$ Zwar hat der EuGH aus einer auf der Betriebsratsrichtlinie 94/45/EG beruhenden Informationspflicht der Mutter auf der Grundlage des Effektivitätsgebots eine Pflicht aller der „Gruppe angehörenden [...] Unternehmen“ abgeleitet, die „Leitung bei der Erfüllung dieser Hauptpflicht zu unterstützen“ und ihr die hierfür „unerlässlichen Informationen [...] zur Verfügung zu stellen“ ${ }^{33}$ Diese Rechtsprechung lässt sich aber nicht auf die Ad-hoc-Informationspflicht übertragen, ${ }^{34}$ da Art. 11 Abs. 1 der Betriebsratsrichtlinie ausdrücklich auch die Gruppe als solche adressiert und so alle Konzerngesellschaften in die Pflicht nimmt. ${ }^{35}$ Im Gegensatz hierzu ist die Ad-hoc-Informationspflicht ausdrücklich nur an die Mutter als den jeweiligen Emittenten gerichtet und zudem durch das Gebot der „unverzüglichen“ Veröffentlichung auf das rechtlich und tatsächlich Mögliche beschränkt. Der Auskunftsanspruch der Mutter gegen die Tochter ist daher keine notwendige Folge aus einer unbegrenzten Ad-hoc-Informationspflicht, sondern umgekehrt beschränkt das Fehlen von Auskunftsansprüchen die kapitalmarktrechtliche Ad-hoc-Infor-

5. Aufl. 2020, $₫ 311$ Rdn. 425 m.w.N.; KoCH, in: Hüffer/Koch, 14. Aufl. 2020, 99 AktG Rdn. 7a; $₫ 311$ AktG Rdn. 36d.

30 KOCH, in: Hüffer/Koch aaO (Fn. 29), §311 AktG Rdn. 36d; SCHÜRNBRAND, ZHR 181 (2017), 357, 367; VERSE, ZHR 175 (2011), 401, 423. AA. jedenfalls zur Erfüllung bestehender gesetzlicher Publizitätspflichten HABERSACK, in FS 25 Jahre WpHG, 2019, S. 217, 233; SEMLER, Leitung und Überwachung der Aktiengesellschaft, 2. Aufl., 1996, S. 300 ff.; U.H. SCHNEIDER/S.H. SCHNEIDER, ZIP 2007, 2061, $2064 \mathrm{f}$.

31 So LG Stuttgart, Beschl. v. 28.2.2017 - 22 AR 1/17 Kap = WM 2017, 1451 Rdn. 195 ff.; VeIL/BrÜGgEMEIER, in: Meyer/Veil/Rönnau, aaO (Fn. 21), \10 Rdn. 23; PFÜlLER, in: Fuchs, aaO (Fn. 18) Rdn. 163; U.H. SCHNEIDER, in: Habersack/Mülbert/ Schlitt, Handbuch der Kapitalmarktinformation, 2. Aufl. 2013, \3 Rdn. 31; SINGHOF, ZGR 2001, 146, 164, 170.

32 VeIL/BrügGEMEIER, in: Meyer/Veil/Rönnau, aaO (Fn. 21), \10 Rdn. 23; ähnlich auch LG Stuttgart, Beschl. v. 28.2.2017 - 22 AR 1/17 Kap = WM 2017, 1451 Rdn. 199.

${ }_{33}$ Zur Europäischen Betriebsratsrichtlinie EUGH, Urt. v. 13.10.2004 - C 440/00, ECLI:EU:C:2004:16 Rz. 59 - Kühne \& Nagel.

34 AA. VeIL/BRÜGGEMEIER, in: Meyer/Rönnau/Veil, (Fn. 21), \$ 10 Rdn. 23.

35 EUGH, Urt. v. 13.10.2004 - C 440/00, ECLI:EU:C:2004:16 Rz. 56 - Kühne \& Nagel. 
mationspflicht der Mutter. ${ }^{36}$ Im faktischen Konzern kann von der Mutter daher mangels Auskunftsanspruchs gegen die Tochter allenfalls verlangt werden, dass sie sich innerhalb der insiderrechtlichen und datenschutzrechtlichen Grenzen aktiv um die gem. $\int \$ 311 \mathrm{ff}$. AktG statthafte Weitergabe der Information von der Tochter bemüht. ${ }^{37}$ Verweigert die Tochter die Weitergabe der Information, scheidet die Ad-hoc-Veröffentlichungspflicht der Mutter mangels objektiver Möglichkeit jedoch aus. ${ }^{38}$

\section{Konzernweite Durchsetzung des Marktmissbrauchsrechts}

Spannungen zwischen Konzern- und Marktmissbrauchsrecht entstehen auch auf Sanktionsebene. Der europäische Gesetzgeber hat als Konsequenz aus der Finanzmarktkrise vor allem mit strengeren Bußgeldregeln reagiert und sich dabei an den als besonders schlagkräftig bekannten kartellrechtlichen Instrumentarien ${ }^{39}$ orientiert. Im Kartellrecht verbinden sich Mutterund Tochtergesellschaft nicht nur zu einer wirtschaftlichen Einheit als Normadressat (siehe B.), sondern bilden auch eine „Haftungsklammer“, ${ }^{40}$ die für Kartellverstöße der Tochter gesamtschuldnerisch mit einem Bußgeld in Höhe von zehn Prozent des Konzerngesamtumsatzes bebußt werden kann. Auf Grundlage von Art. 30 Abs. 2 Buchst. i, j MAR ${ }^{41}$ kann marktmissbräuchliches Verhalten von juristischen Personen und Personengesellschaften gem. $₫ 120$ Abs. 17-22 WpHG mit Bußgeldern bis zu einer Höchstgrenze - je nach Art des Verstoßes - von zwei bis zu 15 Prozent des Jahresgesamtumsatzes bebußt werden. Bei Verstößen einer Tochter- oder Muttergesellschaft ist gem. $₫ 120$ Abs. $23 \mathrm{WpHG}$ für die Berechnung der umsatzabhängigen Bußgeldhöchstgrenze „der jeweilige Gesamtbetrag in

${ }^{36}$ Gegen einen kapitalmarktrechtlichen Auskunftsanspruch bereits HOPT, aaO (Fn. 1), S. 31, 59. Zu Art. 17 MAR BARTMAnN, aaO (Fn. 20), S. 313; BeHN, Ad-hoc-Publizität und Unternehmensverbindungen, 2012, S. $200 \mathrm{ff}$.; HABERSACK, in FS 25 Jahre WpHG, 2019, S. 217, 233 f.; KLÖHN, in: Klöhn, aaO (Fn. 19), Art. 17 MAR Rn. 133. AA. LG Stuttgart, Beschl. v. 28.2.2017 - 22 AR 1/17 Kap = WM 2017, 1451 Tz. 195 ff.; VEIL/ BRÜGGEMEIER, in: Meyer/Rönnau/Veil, (Fn. 21), \$10 Rdn. 23.

37 HABERSACK, in FS 25 Jahre WpHG, 2019, S. 217, 233 f.; KlÖHN, in: Klöhn, aaO (Fn. 19), Art. 17 MAR Rn. 133. Im Rahmen der Wissenszurechnung zu den Anforderungen an das Wissensmanagement SCHÜRNBRAND, ZHR 181 (2017), 357, 369.

38 HABERSACK, in: FS 25 Jahre WpHG, 2019, S. 217, 233; SCHÜRNBRAND, ZHR 181 (2017), 357, 368. AA. VeIL/BRÜGgEMEIER, in: Meyer/Rönnau/Veil, (Fn. 21), §10 Rdn. 23.

39 Zum Ausmaß der Bußgeldsanktionen im Kartellrecht DREHER/KULKA, Wettbewerbs- und Kartellrecht, 10. Aufl. 2018, Rdn. 1759.

40 FÜLLER, in: KölnKomm. z. KartellR, 2016, Art. 101 AEUV, Rdn. 47.

41 Ähnliche Vorschriften finden sich auch in anderen europäischen kapitalmarktrechtlichen Vorschriften, siehe nur Art. 70 Abs. 6 Buchst. f, g MiFID II, Art. 38 Abs. 2 Buchst. d Prospektverordnung; Art. 28b Abs. 1 Buchst. c) Transparenzrichtlinie, und im Datenschutzrecht (siehe Art. 83 Abs. 4 bis 6 DSGVO sowie ErwGr 150 S. 3; hierzu FAUST/ SPITTKA/WYBITUL, ZD 2016, 120; SPINDLER, DB 2016, 937. 
dem Konzernabschluss des Mutterunternehmens maßgeblich, der für den größten Kreis von Unternehmen aufgestellt wird“ (siehe $₫ 120$ Abs. 23 S. 2 WpHG). Art. 30 Abs. 2 UAbs. 2 MAR bringt deutlich zum Ausdruck, dass es nicht auf die tatsächliche Aufstellung, sondern lediglich auf die Pflicht zur Aufstellung eines Konzernabschlusses iSd. Art. 22 der Bilanzrichtlinie 2013/ 34/EU bzw. $\ 290 \mathrm{HGB}$ ankommt. Hierfür genügt gem. $\ 290$ Abs. 1 S. 1 HGB, dass die Gesellschaft unmittelbar oder mittelbar beherrschenden Einfluss auf andere Gesellschaften ausüben kann. ${ }^{42}$

\section{Bußgeldrechtliche Verantwortung der Muttergesellschaft}

Zunächst stellt sich die Frage, ob bei kapitalmarktrechtlichen Verstößen der Tochtergesellschaft diese allein oder - wie im europäischen und deutschen Kartellbußgeldrecht gem. Art. 23 VO 1/2003 $3^{43}$ bzw. $\$ 81$ Abs. 3a GWB ${ }^{44}-\mathrm{da}-$ neben auch die Muttergesellschaft gesamtschuldnerisch mit einem Bußgeld sanktioniert werden kann. Parallel zum gesellschaftsrechtlichen Trennungsprinzip $^{45}$ gilt im Ordnungswidrigkeitenrecht das Rechtsträgerprinzip, wonach nur der jeweilige Rechtsträger, also die zuwiderhandelnde Gesellschaft, bebußt werden kann. ${ }^{46}$ Eine $₫ 81$ Abs. 3a GWB vergleichbare Regelung, wonach gegen Unternehmen, die zum Zeitpunkt eines Kartellverstoßes auf die zuwiderhandelnde Gesellschaft unmittelbar oder mittelbar einen bestimmenden Einfluss ausgeübt haben, eine Geldbuße festgesetzt werden kann, existiert im Marktmissbrauchsrecht nicht. Eine bußgeldrechtliche Konzernverantwortung der Muttergesellschaft für Verstöße ihrer Töchter, an denen Leitungspersonen oder Mitarbeiter der Mutter nicht aktiv mitgewirkt haben, kann daher allenfalls über $₫ 130 \mathrm{OWiG}$ begründet werden, wenn der gesamte Konzern mit einem Unternehmen und die Muttergesellschaft mit dem Unternehmensinhaber iSd. $\$ 130$ OWiG gleichgesetzt werden kann und darüber hinaus eine Aufsichtspflicht der Muttergesellschaft für die Einhaltung des Kapitalmarktrechts in den Tochtergesellschaften besteht. ${ }^{47}$

42 Beschlussempfehlung und Bericht z. MoMiG BT Drucks 16/12407, S. 89 f.

${ }^{43}$ Grundlegend EUGH, Urt. v. 14.7.1972 - C 48/69 = ECLI:EU:C:1972:70, Rz. 136/ 141: Imperial Chemical Industries.

${ }^{44}$ Konsequent hat der EuGH jüngst entschieden, dass der Unternehmensbegriff im Zivilrecht ebenso zu verstehen ist wie im Bußgeldrecht (EUGH, Urt. v. 14.3.2019 - C 724/17 = ECLI:EU:C:2019:204, Rz. 6ff.: Vantaan kaupunki = NZKart 2019, 216 Rdn. 47), sodass die Mutter auch zivilrechtlich für Kartellverstöße der Tochter als Teil der wirtschaftlichen Einheit einzustehen hat (BAUERMEISTER, NZKart 2019, 252, 254).

45 Statt aller WiedemanN, Gesellschaftsrecht, 1980, §4 II 3b S. 214; zur Differenzierung zwischen Unternehmen und Unternehmensträger K. SCHMIDT, Handelsrecht, 6. Aufl., 2014, $₫ 3$ IV 2, Rdn. $44 \mathrm{ff}$.

${ }^{46}$ LÖBBE, ZHR 177 (2013), 518, 541.

47 Hierzu SpOerR, in: Assmann/Schneider/Mülbert, aaO (Fn. 18), $₫ 120$ WpHG Rdn. 418 ff.; Poelzig, aaO (Fn. 6), S. 83, 93 f. Das OLG München hat im Jahr 2014 eine An- 
Gegen ein konzernweites Verständnis des $₫ 130 \mathrm{OWiG}$ spricht, dass $₫ 30$ Abs. 1 Nr. 5 OWiG ausdrücklich die Leitung des „Betriebs oder Unternehmens einer juristischen Person“ erwähnt. Hieran knüpft $₫ 130 \mathrm{OWiG}$ mit den Begriffen des Betriebs und des Unternehmens an und bezieht sich daher ebenfalls auf die zuwiderhandelnde juristische Person, also die Tochtergesellschaft. ${ }^{48}$ Dass allein die Tochtergesellschaft als Inhaberin ihres Unternehmens in Betracht kommt, folgt zudem aus der Funktion des $\int 130$ OWiG, Sanktionslücken zu schließen, die durch die Delegation von unternehmensbezogenen Pflichten auf Mitarbeiter entstehen. ${ }^{49}$ Bei einem Verstoß der Tochtergesellschaft gegen das Marktmissbrauchsrecht kann diese mit einem Bußgeld sanktioniert werden. Eine Sanktionslücke besteht also nicht. ${ }^{50}$ Die materiell-rechtliche Verantwortlichkeit für die Einhaltung des Marktmissbrauchsrechts liegt nicht beim Unternehmen als wirtschaftliche Einheit, sondern bei den Konzerngesellschaften als rechtlich selbständige Personen. Insoweit besteht ein wesentlicher Unterschied zum europäischen Kartellrecht: Dort haben sich die Verfasser der Verträge in Art. 101, 102 AEUV dafür entschieden, das Unternehmen zu adressieren, das dann dementsprechend auch die bußgeldrechtliche Verantwortung für Kartellverstöße übernehmen soll. ${ }^{51}$ Die Entscheidung für eine konzernweite Anwendung

wendbarkeit des $₫ 130 \mathrm{OWiG}$ je nach der konkreten Einflussnahme befürwortet, im konkreten Fall aber eine Anwendung abgelehnt (OLG MÜNCHEN, Urt. v. 23.9.2014 - 3 Ws 599/14 = BB 2015, 2004 Rdn. 16). Der BGH hat diese Frage bisher noch nicht entschieden (siehe aber BGH, Urt. v. 1.12.1981 - KRB 3/79 = GRUR 1982, 244, 247: TransportbetonVertrieb; BGH, Urt. v. 10.8.2011 - KRB 55/10 = BGHSt 57, 193, 200, Rz. 20: Versicherungsfusion). Im Schrifttum ist man sich über die konzernweite Anwendung des $₫ 130$ OWiG uneinig: Dagegen J. KOCH, AG 2009, 564 ff.; KORT, in: Großkomm. z. AktG, 5. Aufl., 2015, § 91 Rdn. 170; ACHENBACH, NZWiSt 2012, 321, 325 ff.; BOSCH, ZHR 177 (2013), 454, 462 ff.; HABERSACK, in: FS Möschel, S. 1175, 1181 f.; HOLLE, Legalitätskontrolle im Kapitalgesellschafts- und Konzernrecht, 2014, S. 405 ff.; KARBAUM, AG 2013, 863, 864 f.; RÖNNAU, ZGR 2016, 277, 289 ff.; v. SCHREITTER, NZKart 2016, 253, 256 ff.; TSCHIERSCHKE, Die Sanktionierung des Unternehmensverbundes, 2013, S. 51 ff.; VERSE, ZHR 175 (2011), 401, 409ff.; dafür Rogall, in: Karlsruher Komm. z. OWiG, 5. Aufl., 2018, \ 130 Rdn. 27; GRUNDMEIER, Rechtspflicht zu Compliance im Konzern, 2011, S. 59 ff.; LÖBBE, ZHR 177 (2013), 518, $543 \mathrm{ff}$.

48 BRETTEL/THOMAS, Compliance und Unternehmensverantwortlichkeit im Kartellrecht, 2016, S. 39.

${ }^{49}$ RegE OWiG, BT Drucks. V/1269, S. 68 f. Siehe auch BGH, Urt. v. 13.4.1994 - II ZR 16/93 = BGHZ 125, 366, 374; ACHENBACH, NZWiSt 2012, 321, 327; BLOME, Rechtsträgerprinzip und wirtschaftliche Einheit, S. 103; BÖSE, in: Graf/Jäger/Wittig, Komm. z. Wirtschafts- und Steuerstrafrecht, 2. Aufl., 2017, \81 GWB Rdn. 8; GRUNDMEIER, aaO (Fn. 50), S. 75; SCHREITTER, NZKart 2016, 253, 260f.; THÜSING, Beschäftigtendatenschutz und Compliance, $\$ 2$ Rdn. 41; TsCHIERSCHKE, aaO (Fn. 50), S. $54 \mathrm{f}$.

${ }^{50}$ Hierzu nur J. KOCH, WM 2009, 1013, 1018.

51 EUGH, Urt. v. 10.4.2014 - C 231/11 P und C 233/11 P = ECLI:EU:C:2014:256, Rz. 44: Siemens Österreich: „Um den Urheber einer Zuwiderhandlung gegen das Wettbewerbsrecht zu bestimmen, dem gem. Art. [101 und 102 AEUV] eine Sanktion auferlegt werden kann, haben sich die Verfasser der Verträge dafür entschieden, den Unterneh- 
des Kartellbußgeldrechts ist damit bereits auf der materiell-rechtlichen Ebene des Kartellverbots gem. Art. 101, 102 AEUV gefallen und insoweit als Bestandteil des europäischen Primärrechts verbindlich. ${ }^{52}$ Eine unmittelbare Bußgeldverantwortung der Muttergesellschaft für das marktmissbräuchliche Verhalten ihrer Töchter lässt sich mithin nicht begründen. Eltern haften im Kapitalmarktrecht - anders als im Kartellrecht - also grundsätzlich nicht für ihre Kinder.

\section{Sanktionierung der Tochtergesellschaft auf Konzernnivean}

Bei einem Verstoß der Tochtergesellschaft kann diese demnach grundsätzlich allein bis zur konzernumsatzabhängigen Bußgeldhöchstgrenze bebußt werden, ohne dass sie die Muttergesellschaft- wie im Kartellrecht ${ }^{53}$ - auf Regress aus der gesamtschuldnerischen Bußgeldhaftung in Anspruch nehmen könnte. ${ }^{54}$ Eltern haften im Kapitalmarktrecht - anders als im Kartellrecht zwar nicht für ihre Kinder. Kinder haften vielmehr allein, aber mit dem Umsatz ihrer Eltern. ${ }^{55}$ Dabei kann es dem europäischen Gesetzgeber nicht darum gehen, die Vermögensmehrung durch den Marktmissbrauch, von der auch die Muttergesellschaft regelmäßig profitiert ${ }^{56}$ bei der Mutter mit abzuschöpfen, denn diese wird gerade nicht mit einem Bußgeld belegt. Mit der Anknüpfung an den Gesamtumsatz soll vielmehr sichergestellt werden, dass sich die Geldbußen im Rahmen der wirtschaftlichen Leistungsfähigkeit des Betroffenen bewegen und größere Unternehmen ebenso empfindlich getroffen werden wie kleinere Unternehmen. ${ }^{57}$ So weist der deutsche Gesetzgeber in den Materialien zur Umsetzung der europäischen Vorgaben darauf hin, dass „,...] der

mensbegriff zu verwenden [...] Nach ständiger Rechtsprechung bezeichnet der Begriff des Unternehmens jede eine wirtschaftliche Tätigkeit ausübende Einheit unabhängig von ihrer Rechtsform und der Art ihrer Finanzierung. [...] Verstößt eine solche wirtschaftliche Einheit gegen die Wettbewerbsregeln, so hat sie nach dem Grundsatz der persönlichen Verantwortlichkeit für diese Zuwiderhandlung einzustehen.". Siehe auch EuGH, Urt. v. 10.9.2009 - C 97/08 P = ECLI:EU:C:2009:536, Rz. 58: KZO.

52 WeCK, NZG 2016, 1374, 1375. Hierzu kritisch HommelHoff, ZGR 2019, 379, 404 ff.; POelZIG, aaO (Fn. 6), S. 83, 87 ff.

${ }^{53}$ EuGH, Urt. v. 10.4.2014 - C 231/11 P bis C 233/11 P = ECLI:EU:C:2014:256, Rz. 67: Siemens Österreich.

${ }^{54}$ So auch kritisch zu $₫ 81$ Abs. 4 S. 3 GWB aF vor der 9. GWB-Novelle KLUSMANN, ZGR 2016, 252, 260f. Positiv hingegen MAUME, ZHR 180 (2016), 358, 384.

55 Vgl. der treffende Titel des Beitrags zu $₫ 81$ Abs. 4 GWB aF KLusmann, in: FS Canenbley, 2012, S. 291.

${ }^{56} \mathrm{Zu}$ diesem Aspekt der bußgeldrechtlichen Inanspruchnahme der Muttergesellschaft im Kartellrecht HOMMELHOFF, ZGR 2019, 379, 407.

57 VeIL, ZGR 2016, 305, 315. Vgl. \81 Abs. 4 GWB aF. BGH, Urt. v. 26.2.2013 - KRB 20/12 = BGHSt 58, 158 Rz. 61 - Grauzementkartell. Siehe ausführlich zu dieser Funktion von Bußgeldhöchstgrenzen ACKERMANN, ZWeR 2010, 329, $338 \mathrm{f}$. 
gesamte Konzern eine größere Wirtschaftskraft besitzt und damit auch höhere Geldbußen möglich sein müssen ". ${ }^{58}$ Ähnlich hat der BGH in der Granzement-Entscheidung zur kartellrechtlichen Konzernumsatzanknüpfung in $\S 81$ Abs. 4 S. 2, $3 \mathrm{GWB}$ aF festgestellt, dass „es für die wirtschaftliche Bewertung entscheidend auf das Unternehmen als wirtschaftliche Einheit ankommt" und sich die „Ahndungsempfindlichkeit und der sich hieraus ergebende Abschreckungseffekt [...] nicht nach den wirtschaftlichen Daten der juristischen Person, für die gehandelt wurde, sondern nach denjenigen des Gesamtunternehmens" bestimmt. ${ }^{59}$

Nach dem EuGH muss „die Härte der Sanktionen der Schwere der mit ihnen geahndeten Verstöße entsprechen [...]", indem sie insbesondere eine wirklich abschreckende Wirkung gewährleistet, zugleich aber den allgemeinen Grundsatz der Verhältnismäßigkeit wahrt. ${ }^{60} \mathrm{Zu}$ berücksichtigen ist dabei insbesondere auch die Wirtschaftskraft der bebußten Person. Bußgelder sollen abschrecken, aber nicht vernichten. ${ }^{61}$ Anders als es der BGH auf Grundlage der kartellrechtlichen Rechtsprechung des EuGH postuliert, ${ }^{62}$ kann es im Marktmissbrauchsrecht für die Ahndungsempfindlichkeit grundsätzlich nicht auf die Perspektive der wirtschaftlichen Einheit ankommen, da nicht das Unternehmen, sondern natürliche und juristische Personen Adressaten des Marktmissbrauchsrechts und damit Täter sind. Ob und inwieweit die zuwiderhandelnde Tochtergesellschaft auf die Wirtschaftskraft des Konzerns zugreifen kann, hängt letztlich von den konkreten Umständen des Einzelfalls ab und zwar vor allem davon, ob es sich um einen Vertragskonzern oder einen faktischen Konzern handelt.

58 Begr. RegE TranspRLÄndRLUG, BT-Drucks. 18/5010, S. 53.

${ }^{59}$ So auch OLG DÜSSELDORF, Urt. v. 26.6.2009 - VI-2a Kart 2 - 6/08 OWi = juris, Rz. 618 (der juristischen Person als Teil eines Konzerns „wächst ein erweiterter Verhal-

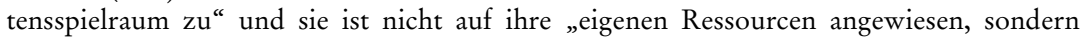
kann auf die Wirtschaftskraft des Gesamtkonzerns zurückgreifen“.

60 EuGH, Urt. v. 16.7.2015 - C 255/14 = ECLI:EU:C:2015:475, Rz. 20 ff. = EuGRZ 2015, 497 mit Verweis auf EuGH, Urt. v. 25.4.2003 - C 81/12 = EU:C:2013:275, Rz. 63: Asociația Accept; EuGH, Urt. v. 27.3.2014 - C 565/12 = EU:C:2014:190, Rz. 45: LCL Le Crédit Lyonnais. Teilweise wird auch auf den Schuldgrundsatz gem. \49 EuGrCh abgestellt (so etwa ACHENBACH, wistra 2018, 13, 17; zum Kartellrecht auch THOMAS, in: FS Möschel, 2011, S. 675, 688). Ob und inwieweit der Schuldgrundsatz auf europäische Bußgeldregelungen anwendbar ist, ist jedoch umstritten (näher hierzu KINDHÄUSER/MEYER, in: Jaeger/Kokott/Pohlmann/Schroeder, Frankfurter Komm. z. Kartellrecht, 93. Lieferung 04/2019, Bußgeldrechtliche Folgen des Art. 101 AEUV, Rdn. 136 m.w.N.). Der EuGH beschränkt die Geltung des Schuldgrundsatzes auf strafrechtliche Sanktionen und lehnt den strafrechtlichen Charakter von Verwaltungssanktionen ab (EuGH, Urt. v. 11.7.2002 C 210/00, Slg. 2002, I-6453, Rz. 35 ff.: Champignon Hofmeister). Allgemein zur Anwendbarkeit der EuGrCh auf $\int 120 \mathrm{WpHG}$ BRAND/HOTZ, ZIP 2017, 1450, 1454; GAEDE, wistra 2017, 41, 46f.; MÖLLERS/HERZ, JZ 2017, 445, 449 f.

${ }^{61}$ PALZER, NZI 2012, 67, 72.

62 EuGH, Urt. v. 10.9.2009 - C 97/08 P = ECLI:EU:C:2009:536, Rz. 77: Akzo Nobel. Auf diesen Aspekt ebenfalls hinweisend ACHENBACH, wistra 2018, 13, 17. 


\section{a) Vertragskonzern}

Im Vertragskonzern steht der Tochter regelmäßig ein Verlustausgleichsanspruch gem. \302 Abs. 1 AktG zu, sodass sie das Bußgeld der Muttergesellschaft in Rechnung stellen kann. ${ }^{63}$ Anders als das Kartellrecht sieht das Marktmissbrauchsrecht damit zwar keinen echten Haftungsdurchgriff vor, da die Muttergesellschaft nicht persönlich im Außenverhältnis bebußt werden kann (III.1.). Es handelt sich aber um einen Berechnungsdurchgriff, ${ }^{64}$ der einer unmittelbaren eigenen Bußgeldverantwortlichkeit der Muttergesellschaft im Außenverhältnis wirtschaftlich gleichkommt. Dies geschieht indes unabhängig von einem eigenen Fehlverhalten der Muttergesellschaft; die Anknüpfung der Bußgeldhöchstgrenze an den Konzernumsatz basiert allein auf der Konzernbeziehung. ${ }^{65}$ Die Muttergesellschaft wird im Vertragskonzern also ohne eigenes vorwerf- und vermeidbares Verhalten indirekt und pauschal durch das konzernumsatzabhängige Bußgeld über das Innenverhältnis zur Tochtergesellschaft mit in die Verantwortung genommen. ${ }^{66} \mathrm{Da}$ die Mutter auch am Gewinn aus dem marktmissbräuchlichen Verhalten partizipiert, erscheint dies im Hinblick auf den Abschöpfungsanteil der Geldbuße konsequent, mit Rücksicht auf den Ahndungsanteil der Geldbuße aber ohne vorwerfbares Verhalten der Muttergesellschaft problematisch. ${ }^{67}$

$\mathrm{Zu}$ bedenken ist darüber hinaus, dass der Anspruch der Tochtergesellschaft auf Verlustausgleich erst mit dem Bilanzstichtag fällig wird. ${ }^{68}$ Ist das Bußgeld aber - wie in der überwiegenden Zahl der Fälle zu erwarten - mehr als drei Wochen vor dem Bilanzstichtag zu zahlen, kommt der Verlustausgleich zu spät und kann die Zahlungsunfähigkeit der Tochtergesellschaft gem. \17 InsO durch ein konzernumsatzabhängiges Bußgeld in der Regel nicht mehr verhindern. ${ }^{69}$ Die Funktion der Bußgeldhöchstgrenze, abschreckende, aber zugleich verhältnismäßige Sanktionen zu ermöglichen, liefe

${ }^{63}$ Kremer/Klahold, in: Krieger/Schneider, Handbuch Managerhaftung, 3. Aufl., 2017, Rdn. 25.13; VERSE/WIERSCH, ZWeR 2015, 21, 26. Zur vergleichbaren Vorschrift des \81 Abs. 4 S. 3 GWB idF. 2005 vor der 9. GWB-Novelle BUNTSCHECK, in: FS Bechtold, 2006, S. 81, 87; ihm folgend ABERLE, Sanktionsdurchgriff und wirtschaftliche Einheit im deutschen und europäischen Kartellrecht, 2013, S. 217.

${ }^{64} \mathrm{Zu}$ dem Begriff BITTER, in: Scholz, Komm. z. GmbHG, 12. Aufl., 2018, §13 Rdn. 74a; $188 \mathrm{ff}$.

${ }^{65}$ VEIL, ZGR 2016, 305, 317; ACHENBACH, wistra 2018, 11, 17.

${ }^{66}$ Kritisch hierzu Poelzig, aaO (Fn. 6), S. 83, 109 f.

${ }^{67}$ Zur Differenzierung zwischen Abschöpfungs- und Ahndungsanteil kartellrechtlicher Geldbußen HOMMELHOFF, ZGR 2019, 379, 406 f. Insoweit kritisch auch ACHENBACH, wistra 2018, 11, $17 \mathrm{f}$.

68 BGH, Urt. v. 11.10.1999 - II ZR 120/98 = BGHZ 142, 382, 385 f. Hierzu statt aller Altmeppen, in: MüKo-AktG, aaO (Fn. 29), \$ 302 Rdn. 73 f.

69 Thomas, aaO (Fn. 60), S. 675, 689. 
leer. ${ }^{70}$ Auf die Wirtschaftskraft der Muttergesellschaft kann daher (nur) abgestellt werden, wenn man - entgegen der h.M. ${ }^{71}$ - die Muttergesellschaft zu Abschlagszahlungen auch während des laufenden Geschäftsjahres verpflichtet, um die Lebensfähigkeit der abhängigen Gesellschaft zu erhalten. ${ }^{72}$

\section{b) Faktischer Konzern}

Im faktischen Konzern hat die Tochter hingegen grundsätzlich keine Möglichkeit, auf die finanziellen Ressourcen der Muttergesellschaft zuzugreifen. ${ }^{73}$ Die Nachteilsausgleichspflicht der herrschenden Gesellschaft beschränkt sich gem. $₫ 317$ Abs. 1 AktG auf die Fälle, in denen sie das abhängige Unternehmen zu nachteiligen Geschäften veranlasst. Sofern die Mutter an dem Verstoß nicht ursächlich mitgewirkt hat, kommt daher ein Regressanspruch der Tochter- gegen die Muttergesellschaft - etwa gem. $\S 317$ Abs. 1 AktG oder gem. $₫ 826$ BGB in Form der Existenzvernichtungshaftung ${ }^{74}-$ nicht in Betracht. Dass die Muttergesellschaft uU. auch ohne gesetzliche Verpflichtung bereit ist, einen Teil der Geldbuße zu übernehmen, um die Insolvenz der Tochter zu vermeiden, kann für die Wirtschaftskraft und damit für die Ahndungsempfindlichkeit der Tochtergesellschaft nicht ausschlaggebend sein. ${ }^{75}$ Im faktischen Konzern scheidet damit ein Rückgriff bei der Muttergesellschaft aus, so dass die Tochtergesellschaft das auf Konzernniveau berechnete Bußgeld gem. $\ 120 \mathrm{WpHG}$ allein zu tragen hat. Das drohende Bußgeld kann daher theoretisch allein aufgrund der Konzernzugehörigkeit deutlich höher ausfallen als gegenüber nicht konzernierten Gesellschaften und damit die Ahndungsempfindlichkeit der Tochter unverhältnismäßig überstrapazieren.

70 So auch zur parallelen Problematik im Kartellrecht vor der 9. GWB-Novelle BRETTEL/THOMAS, ZWeR 2009, 25, 59.

71 BGH, Urt. v. 19.9.1988 - II ZR 255/87 = BGHZ 105, 168 = BGH AG 1989, 27, 29 : Hamburger Stahlwerke (kein „rechtlich unzweifelhafter Anspruch“); KOPPENSTEINER, in: KölnKomm. z. AktG, 3. Aufl., 2004, $\$ 302$ Rdn. 57; HIRTE, in: Großkomm. z. AktG, 4. Aufl., 2013, § 302 Rdn. 62; KRIEGER, in: Münch. Hdb. AG, 4. Aufl., 2015, \ 70 Rdn. 75; PASCHOS, in: Henssler/Strohn, Komm. z. Gesellschaftsrecht, 4. Aufl., 2019, \302 AktG Rdn. 15; SERVATIUS, in: Grigoleit, AktG, Kommentar, 2013, §302 Rdn. 14; DeILmanN, in: Hölters, Komm. z. AktG, 3. Aufl., 2017, $₫ 302$ Rdn. 23; LIEBSCHER, ZIP 2006, 1221 f.; MERTENS, ZGR 1984, 542, $551 \mathrm{f}$.

72 So Altmeppen, in: MüKo-AktG, aaO (Fn. 29), § 302 Rdn. 38, 74; DERs., DB 1999, 2453, 2455 f.; PRIESTER, ZIP 1989, 1301, 1307 f.; U.H. SCHNEIDER, ZGR 1984, 497, 534 f.; EMMERICH, in: Emmerich/Habersack, Komm. z. Aktien- und GmbH-Konzernrecht, 8. Aufl., 2016, $₫ 302$ AktG Rdn. 41; VeIL, in: Spindler/Stilz, Komm. z. AktG, 4. Aufl., 2019, \ 302 Rdn. 23.

${ }^{73}$ So zu $₫ 81$ Abs. 4 S. 3 GWB aF vor der 9. GWB-Novelle THOmas, in: FS Möschel, 2011, S. 675, $688 \mathrm{f}$.

${ }^{74}$ Zur Existenzvernichtungshaftung als Innenhaftung des Gesellschafters gegenüber der GmbH BGH, Urt. v. 16.7.2007 - II ZR 3/04 = BGHZ 173, 246, 257 f.: Trihotel.

${ }^{75} \mathrm{Zu} \S 81$ Abs. 4 S. 3 GWB aF ThOMAS, in: FS Möschel, 2011, S. 675, 689. 


\section{c) Zwischenergebnis}

Indem die Tochter allein auf Konzernniveau bebußt werden kann, ohne dass die Mutter für das Bußgeld im Außenverhältnis gesamtschuldnerisch mithaftet, ähnelt das Marktmissbrauchsrecht dem deutschen Kartellbußgeldrecht vor der 9. GWB-Novelle gem. $₫ 81$ Abs. 4 S. 2 GWB aF. ${ }^{76}$ Der Weg des deutschen Kartellgesetzgebers, diesen „Konstruktionsfehler “77 durch Einführung der gesamtschuldnerischen Bußgeldverantwortung der Muttergesellschaft gem. $\ 81$ Abs. 3 a GWB zu beseitigen, ${ }^{78}$ ist im Marktmissbrauchsrecht jedoch versperrt: Denn anders als im Kartellrecht korrespondiert die konzernweite Bemessungsgrundlage der Bußgeldhöchstgrenze hier nicht mit der materiell-rechtlichen Verantwortlichkeit. Wird die bußgeldrechtliche Konzernverantwortung im europäischen Kartellrecht vor allem damit begründet, dass das Unternehmen als wirtschaftliche Einheit Adressat des Kartellrechts ist, ${ }^{79}$ erscheint die Rechtfertigung einer konzernweiten Verantwortung im europäischen Marktmissbrauchsrecht mit Blick auf das gesellschaftsrechtliche Trennungsprinzip (noch) schwieriger, da hier natürliche und juristische Personen und nicht Unternehmen als wirtschaftliche Einheit adressiert werden (siehe II.).

\section{Bußgeldbemessung}

Um eine verhältnismäßige Sanktionierung in Konzernverhältnissen sicherzustellen, gewinnen daher die Kriterien der Bußgeldbemessung im Einzelfall erheblich an Bedeutung. ${ }^{80}$ So weisen auch die Erwägungsgründe ausdrücklich darauf hin, dass die Bußgeldhöchstgrenzen nur bei schweren Verstößen ausgeschöpft werden sollen. ${ }^{81}$ Demnach hat die BaFin bei der Bußgeldbemessung ua. auch ein angemessenes Verhältnis zur wirtschaftlichen Leistungsfähigkeit des Bußgeldadressaten zu wahren (vgl. \17 Abs. 3 S. $2 \mathrm{OWiG}){ }^{82}$ Maßgeblich ist - anders als für die Bestimmung der Bußgeld-

${ }^{76}$ Kritisch insoweit auch zu $₫ 81$ Abs. 4 S. 3 GWB aF THOMAS, in: FS Möschel, S. 675, 687 ff.; KlUSMAnN, in: FS Canenbley, 2012, S. 291, 304; AbERLE, aaO (Fn. 68), S. 233 („missglückte Vorschrift“).

77 THOMAS, in: FS Möschel, S. 675, 687.

78 Begr. RegE zur 9. GWB-Novelle, BT-Drucks. 18/10207, S. 85: Stellt man bei der Bußgeldbemessung auf die Umsätze des Gesamtunternehmens ab, wäre es „inkonsequent, bei der Verhängung und Vollstreckung der so bemessenen Geldbuße allein auf die nach außen handelnde Gesellschaft zuzugreifen und die Konzernstrukturen unberücksichtigt zu lassen.“

79 EuGH, Urt. v. 10.4.2014 - C 231/11 P und C 233/11 P = ECLI:EU:C:2014:256, Rz. 44: Siemens Österreich.

${ }^{80}$ Daher gegen verfassungsrechtliche Bedenken SPOERR, in: Assmann/Schneider/ Mülbert, aaO (Fn. 18), \120 WpHG Rdn. 384.

${ }^{81}$ Siehe etwa ErwGr 57 S. 4 MAR.

82 NARTOWSKA/WALLA, NZG 2015, 977, 983. 
höchstgrenze gem. $₫ 120$ Abs. $23 \mathrm{WpHG}$ und anders auch als in $\ 81 \mathrm{Abs}$. 4a GWB - keine Konzernbetrachtung, sondern zu berücksichtigen ist die Finanzkraft der verantwortlichen Person, „wie sie sich zum Beispiel aus dem Gesamtumsatz einer juristischen Person [...] ablesen lässt“ (Art. 31 Abs. 1 Buchst. c) MAR). Dem trägt die BaFin dadurch Rechnung, dass sie bei der Bußgeldbemessung auf die tatsächliche wirtschaftliche Leistungsfähigkeit der Tochter abstellt. ${ }^{83}$ Hierbei ist zu berücksichtigen, ob und inwieweit die zuwiderhandelnde Tochtergesellschaft tatsächlich auf die Wirtschaftskraft des Konzerns zugreifen kann, indem ihr beispielsweise ein Anspruch gegen die Mutter auf Verlustausgleich oder auf Abschlagszahlungen zusteht. Freiwillige Leistungen der Muttergesellschaft sollten hingegen außer Betracht bleiben.

\section{Ergebnis}

1. Damit ist die von Klaus J. Hopt aufgeworfene Frage nach dem Verhältnis von Kapitalmarktrecht und Konzernrecht ${ }^{84}$ für das europäische Marktmissbrauchsrecht weder strikt zugunsten der einen noch der anderen Regelungsmaterie zu beantworten. Spannungen sind durch eine wohl abgewogene und abgestimmte Anwendung unter Berücksichtigung der kapitalmarktrechtlichen Ziele und der Konzernierungsfreiheit aufzulösen. ${ }^{85}$

2. Im Rahmen der Ad-hoc-Publizitätspflicht gem. Art.17 MAR bedeutet dies, dass die Muttergesellschaft sie unmittelbare betreffende kursrelevante Informationen in Tochtergesellschaften nur veröffentlichen muss, soweit sie ihr nach konzernrechtlichen Grundsätzen zugänglich sind. Art. 17 MAR begründet keinen kapitalmarktrechtlichen Auskunftsanspruch. Hierfür spricht vor allem, dass das Marktmissbrauchsrecht - anders als das Kartellrecht juristische Personen und nicht wirtschaftliche Einheiten adressiert und daher die gesellschaftsrechtlichen Grenzen grundsätzlich akzeptiert.

3. Kinder haften für marktmissbräuchliches Verhalten allein, aber mit Einführung der konzernumsatzabhängigen Bußgeldhöchstgrenzen uU. mit

${ }^{83}$ BaFin, WpHG-Bußgeldleitlinien II, BaFin Journal März 2017, 15, 19: Bei einem Rechtsverstoß einer Tochtergesellschaft ist „zwar bei der Bestimmung des Bußgeldrahmens auf den Gesamtumsatz des Konzerns abzustellen, in Schritt drei der Stufe zwei jedoch in der Regel auf die aktuelle wirtschaftliche Leistungsfähigkeit der Tochtergesellschaft" (VON BUTTLAR/BRÜCK, BaFin Journal März/2017, S. 19).

${ }^{84}$ HOPT, aaO (Fn. 1), S. 31, 33.

${ }^{85}$ Zur primärrechtlichen Begründung des Bedürfnisses nach „praktischer Konkordanz“ im Verhältnis von Konzernrecht und europäischem Wettbewerbsrecht HOMMELHOFF, ZGR 2019, 379, 408. Zum Verhältnis von Aktienrecht und Marktmissbrauchsrecht HABERSACK, in: FS 25 Jahre WpHG, 2019, S. 217, 234. 
dem Umsatz ihrer Eltern. Das hat im Vertragskonzern einen Berechnungsdurchgriff auf die Muttergesellschaft zur Folge, sodass diese bei Fehlverhalten der Tochter ohne Rücksicht auf ein eigenes Fehlverhalten im Innenverhältnis auf Konzernniveau in Anspruch genommen werden kann. Im faktischen Konzern führt die Konzernumsatzanknüpfung hingegen dazu, dass die Tochtergesellschaft ohne Möglichkeit zum Regress weit über ihre eigene wirtschaftliche Leistungsfähigkeit hinaus auf Konzernniveau bebußt werden kann. Um gleichwohl eine verhältnismäßige Sanktionierung unter Berücksichtigung der konzernrechtlichen Grenzen sicherzustellen, ist die wirtschaftliche Leistungsfähigkeit der zuwiderhandelnden Tochtergesellschaft bei der Bemessung des Bußgeldes im konkreten Einzelfall ausreichend zu berücksichtigen. 